Unfallchirurg $2022 \cdot 125: 3-4$

https://doi.org/10.1007/s00113-021-01126-z Angenommen: 9. Dezember 2021

Online publiziert: 20. Dezember 2021

(C) The Author(s), under exclusive licence to Springer Medizin Verlag GmbH, ein Teil von Springer Nature 2021

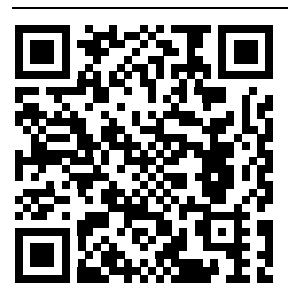

QR-Code scannen \& Beitrag online lesen

\title{
Der Unfallchirurg 2022
}

\section{Ein besonderes Jahr für unsere Zeitschrift}

\author{
Anna Sittig ${ }^{1}$ Christian Krettek ${ }^{2}$. Thomas Mittlmeier ${ }^{3}$ \\ 'Redaktion Der Unfallchirurg, Springer Medizin Verlag GmbH, Heidelberg, Deutschland \\ ${ }^{2}$ Medizinische Hochschule Hannover, Hannover, Deutschland \\ ${ }^{3}$ Klinik für Unfall-, Hand- und Wiederherstellungschirurgie, Universitätsmedizin Rostock, Rostock, \\ Deutschland
}

Liebe Leserinnen und Leser,

mit dieser ersten Ausgabe in 2022 heißen wir Sie in einem besonderen Jahr willkommen. Die Deutsche Gesellschaft für Unfallchirurgie (DGU) und Der Unfallchirurg feiern gemeinsam Jubiläum! Während die DGU ihren 100. Geburtstag begeht, läutet Der Unfallchirurg mit dieser Ausgabe seinen 125. Band ein. Über die bewegte gemeinsame Geschichte von Gesellschaft und Verlag, und warum wir 2022 ein Jubiläum feiern, obwohl Der Unfallchirurg 1894 erstmals publiziert wurde, können Sie im Beitrag von Herrn Prof. Dr. Hans Zwipp in dieser Ausgabe lesen.

Im Lauf ihrer langen Geschichte hat Ihre Zeitschrift bereits viele Gestalter begrüßt und verabschiedet, so geschieht dies auch in diesem Jahr. Wir verabschieden uns von Herrn Prof. Dr. Wolf Mutschler, der die Zeitschrift seit 1999 herausgegeben und geprägt hat. Für sein Engagement sind wir ihm unendlich dankbar und würdigen seine Verdienste in einem weiteren Editorial in dieser Ausgabe.

Die herausgeberische Betreuung der Rubriken „Originalien", „Kasuistiken" und „Kasuistiken kompakt" organisieren wir aus diesem Anlass neu und begrüßen hier neue Gesichter, die bereits in anderer Art und Weise an der Zeitschrift mitgearbeitet haben. Herr Prof. Dr. Hans Polzer und Herr Prof. Dr. Ben Ockert werden die Rubriken in bewährter Weise weiterbetreuen, künftig unterstützt von Herrn PD Dr. Tobias Helfen und Herrn Prof. Dr. Carl Neuerburg. Wir freuen uns, die Beiträge dieser Rubriken dem jungen Team in die Hände zu legen, danken für ihr Engagement und wünschen (weiterhin) viel Freude bei der Mitgestaltung von Der Unfallchirurg.

Einen tragischen Abschied mussten wir bereits im Juli 2021 hinnehmen: Prim. Dr. Thomas Neubauer, Vertreter der ÖGU im Herausgeberboard von Der Unfallchirurg und Rubrikherausgeber für das „Berufspolitische Forum" wurde nach kurzer schwerer Krankheit aus dem Leben gerissen und hinterlässt eine große Lücke. Seiner Familie sowie den Menschen in seinem privaten und beruflichen Umfeld sprechen wir auf diesem Weg unser herzliches Beileid aus. Mit der Umsetzung des Leitthemas "Infektionen des Bewegungsapparates" in dieser Ausgabe, das er vorgeschlagen und geplant hat, möchten wir seiner gedenken und bedanken uns für die Zeit, in der wir fruchtbar zusammenarbeiten durften. Im Herausgeberboard von Der Unfallchirurg wird ihm Prim. Prof. Dr. Christian Kammerlander nachfolgen. Wir freuen uns, die Verbindung zu den Kolleginnen und Kollegen in Österreich aufrechtzuerhalten und zu vertiefen.

\section{》) Gut aufgestellt, wünschen wir} Ihnen, liebe Leserinnen und Lesern, einen guten Start in das Jahr 2022

Bereits seit 01.06.2021 verstärkt Herr Prof. Dr. Dr. Volker Alt als Herausgeber die Rubrik "CME Zertifizierte Fortbildung". Die von unseren Leserinnen und Lesern hochgeschätzte Rubrik ist wichtiges Rückgrat von Der Unfallchirurg, und wir danken Herrn Prof. Alt und den anderen Rubrikherausgebern herzlich für ihr stetiges Engagement für die Fort- und Weiterbildung der Kolleginnen und Kollegen. 


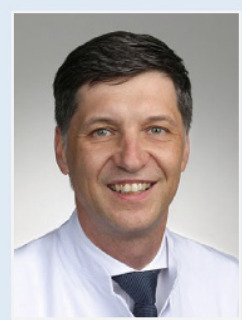

Prof. Dr. Dr. Volker Alt Klinik und Poliklinik für Unfallchirurgie, Universitätsklinikum Regensburg

CME Zertifizierte Fortbildung

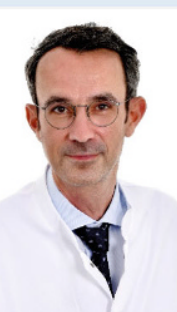

Prof. Dr. Stephan Sehmisch Klinik für Unfallchirurgie, Medizinische Hochschule Hannover

Leitthema

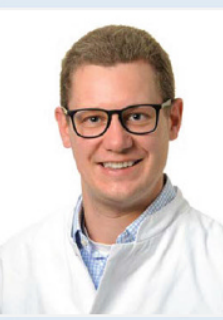

Prof. Dr. Carl Neuerburg MUM -

Muskuloskelettales Universitätszentrum München

Originalien

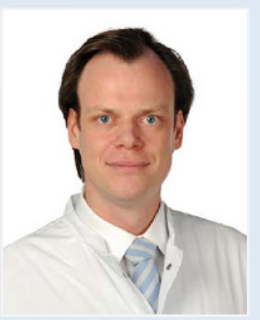

PD Dr. Tobias Helfen MUM Muskuloskelettales
Universitätszentrum München

Kasuistiken \& Kasuistiken kompakt

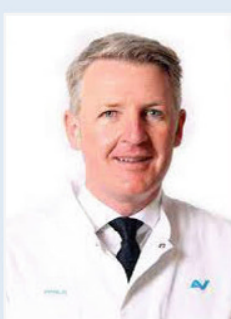

Prof. Dr. Christian Kammerlande AUVA-Unfallkrankenhaus Steiermark, Graz

Berufspolitisches Forum

Abb. 1 \ Herzlich willkommen im Herausgeberboard von Der Unfallchirurg. (Mit freundlicher Genehmigung der gezeigten Personen)

In guter Tradition wird die Rubrik „Leitthema“ seit 1996 in Hannover geführt. Nachdem Herr Prof. Dr. Harald Tscherne diese Aufgabe an seinen Amtsnachfolger Herrn Prof. Dr. Christian Krettek übergeben hat, begrüßt dieser nun seinerseits seinen Amtsnachfolger an der $\mathrm{MHH}$, Herrn Prof. Stephan Sehmisch, zur Mitarbeit in dieser Rubrik. Wir freuen uns und sind dankbar, dass die Betreuung des Leitthemas weiterhin fest in "Hannoveraner Hand" und in der $\mathrm{MHH}$ verankert ist.

Einen Überblick über die neuen Herausgeber von Der Unfallchirurg finden Sie in - Abb. 1 und, in dieser Weise gut aufgestellt, wünschen wir Ihnen, liebe Leserinnen und Lesern, einen guten Start in das Jahr 2022! Bleiben Sie uns gewogen und geben Sie uns gerne jederzeit Feedback zu "Ihrer" Fachzeitschrift; wir sind für jede Anregung dankbar.

Mit herzlichen Grüßen

Christian Krettek, Thomas Mittlmeier, Anna Sittig

\section{Korrespondenzadresse}

\section{Anna Sittig}

Redaktion Der Unfallchirurg, Springer Medizin Verlag GmbH

Tiergartenstr. 17, 69121 Heidelberg,

Deutschland

anna.sittig@springer.com
Interessenkonflikt. A. Sittig ist Redakteurin der Zeitschrift Der Unfallchirurg. C. Krettek und T. Mittlmeier geben an, dass kein Interessenkonflikt besteht.
MED UPDATE SEMINARE 2022

Ortho Trauma Update 2022

13. Orthopädie-UnfallchirurgieUpdate-Seminar

04.-05. März 2022

Berlin und Livestream

\section{Wiss. Leitung:}

Prof. Dr. Ralph Gaulke, Hannover

Prof. Dr. Klaus-Peter Günther, Dresden

Unter der Schirmherrschaft der BVOU/DGSP

www.ortho-trauma-update.com

Auskunft für alle Update-Seminare: med update $\mathrm{GmbH}$

www.med-update.com

Tel.: 0611 - 736580

info@med-update.com

\section{medupdate}

